\title{
Dielectric Function of Native Oxide on Ion-Implanted GaAs
}

\author{
M. Kulik ${ }^{a, *}$, W. RzodKIEwicz ${ }^{b}$, Ł. Gluba ${ }^{a}$ And A.P. Kobzev ${ }^{c}$ \\ ${ }^{a}$ Institute of Physics, Maria Curie-Skłodowska University, pl. M. Curie-Skłodowskiej 1, 20-031 Lublin, Poland \\ ${ }^{b}$ Institute of Electron Technology, al. Lotników 32/46, 02-668 Warszawa, Poland \\ ${ }^{c}$ Frank Laboratory of Neutron Physics, Joint Institute for Nuclear Research, Joliot-Curie 6 \\ 141980 Dubna, Moscow region, Russia
}

\begin{abstract}
The main aim of the reported investigations is the influence of ion implantation on formation of native oxide layers and their optical spectra. Silicon implanted (100)-oriented GaAs crystalline wafers were used as substrates. The samples have been implanted with $\mathrm{Ne}^{+}, \mathrm{Al}^{+}, \mathrm{Ar}^{+}$, or $\mathrm{In}^{+}$ions at energies of 100, 120, 150, and $250 \mathrm{keV}$, respectively. The implantations were carried out at a fluence of $1 \times 10^{16} \mathrm{~cm}^{-2}$ at $300 \mathrm{~K}$. The refraction index spectral dependence for native oxide was approximated using the Cauchy equations. The dielectric function spectra of the native oxide layers on GaAs implanted with different ions have been obtained by variable angle spectroscopic ellipsometer in the 250-900 $\mathrm{nm}$ range using complementary information from the Rutherford backscattering/nuclear reactions measurements. The investigations showed that both real and imaginary parts of the dielectric function increase with mass of the ion species used for implantation.
\end{abstract}

DOI: 10.12693 /APhysPolA.123.956

PACS: 61.72.uj, 78.20.Ci, 78.20.-e

\section{Introduction}

Oxide layers on the surface of $\mathrm{A}^{\mathrm{III}}-\mathrm{B}^{\mathrm{V}}$ semiconductors play an important role in the production of electronic circuits [1]. Ion implantation is a convenient and precise method of fabrication of electronic elements [1-4]. However, due to its physical character this process is accompanied by formation of disordered layers. The investigations of defect formation in the subsurface layers of GaAs implanted with $\mathrm{Ar}^{+}, \mathrm{Si}^{+}$, and $\mathrm{Se}^{+}$ions were presented in [1]. The defects concentration was examined as a function of ion energy, ion current density and fluence. These dependences were investigated for the ion energies from $200 \mathrm{keV}$ to $10 \mathrm{MeV}$ with the fluences varying between $1 \times 10^{12} \mathrm{~cm}^{-2}$ and $1 \times 10^{15} \mathrm{~cm}^{-2}$. The results have shown that the ion mass and energy influence on defective layers formation. The thickness of the disordered layers was measured using the Rutherford backscattering spectrometry with channeling (RBS/C) method [3].

Ion implantation influences formation of native oxide layers on the irradiated semiconductor surfaces exposed to ambient air $[1,2]$. The damage investigations and optical properties of silicon layers implanted by $150 \mathrm{keV}$ $\mathrm{Ne}^{+}$and $800 \mathrm{keV} \mathrm{Ar}{ }^{+}$ions were presented in [4]. The authors used spectroscopic ellipsometry (SE) and Rutherford backscattering/channeling (RBS/C) techniques, and the samples were described by a theoretical model. The proposed optical model consisted of the following components: native oxide $\mathrm{SiO}_{2}$ layer, thin amorphous silicon layer, slightly damaged layer and substrate. There were obtained the thicknesses of all layers. It was observed that with the increasing ion fluence the thickness of native oxide increases. The same effect was investigated for

*corresponding author; e-mail: mkulik@hekt or.umcs.lublin.pl
GaAs implanted with $\mathrm{Ar}^{+}, \mathrm{Al}^{+}$, and $\mathrm{Xe}^{+}$ions [5]. In that study a single-wave ellipsometry was used. The measurements were performed at the wavelength $632.8 \mathrm{~nm}$ and it was found that the refraction index of the native oxide depends on ion fluence.

The influence of low level radiation damage and conditions of implantation on the optical properties of semiconductors has been reported in [6]. The near-edge optical investigations were carried out for low fluence $\mathrm{N}^{+}$ion implanted GaAs at $80 \mathrm{~K}$. It was reported that the point defects influence on an exponential tail of the fundamental absorption edge. The correlations between refractive index and radiation damage in the implanted layers have been investigated [6]. SE and RBS/C studies of the influence of implantation temperature on the dielectric function were performed on hot-implanted GaAs [7]. It was confirmed that the optical models used in the SE interpretation works well for the subsurface implanted layers. The investigations of native oxides on $\mathrm{GaAs}$ were reported [8], using the methods of SE and RBS/NR (nuclear reactions) for GaAs irradiated with $\mathrm{In}^{+}$ion beam.

In the present work silicon implanted (SI) GaAs wafers have been implanted with the ions of $\mathrm{Ne}, \mathrm{Ar}, \mathrm{Al}$ or In at the same ion fluence. The refraction index spectral dependence of native oxides was approximated by the Cauchy equation. The dielectric function spectra of the native oxide layers on GaAs implanted with different ions were determined by the variable angle spectroscopic ellipsometer (VASE).

\section{Experimental}

The SI (100) GaAs wafers were irradiated with $\mathrm{Ne}^{+}$, $\mathrm{Al}^{+}, \mathrm{Ar}^{+}$or $\mathrm{In}^{+}$ions at room temperature (RT) using the UNIMAS ion implanter of the Maria Curie-Skłodowska University $[9,10]$. The ion energies were: 100, 120, 150, and $250 \mathrm{keV}$, respectively. For each implantation the 
same ion fluence value of $1 \times 10^{16} \mathrm{~cm}^{-2}$ was chosen and the current density did not exceed $1 \mu \mathrm{A} \mathrm{cm}{ }^{-2}$ to avoid dynamic annealing. Following ion implantation the samples have been exposed to ambient air for 1 month. All samples before and after ion implantation were investigated using the methods of RBS/NR and SE. The ellipsometric angles $\Psi(\lambda)$ and $\Delta(\lambda)$ spectra were collected in the wavelength region from 250 to $900 \mathrm{~nm}$ by VASE of Woollam [11]. The measurements were made at the incidence angles: $65^{\circ}, 70^{\circ}$, and $75^{\circ}$. Next, the samples were analyzed with the RBS/NR technique. The spectra were collected for the incident $\mathrm{He}^{+}$beam of energy in the range from 3.035 to $3.060 \mathrm{MeV}$. The resolution of semiconductor detector was $12 \mathrm{keV}$ and the scattering angle was equal to $170^{\circ}$. The latter experiment was performed in JINR in Dubna.

\section{Results and discussion}

The RBS/NR study was carried out in order to verify the presence of oxygen-enriched layers on surfaces of the ion implanted GaAs samples. In Fig. 1 the typical RBS/NR spectrum measured for the sample irradiated with $150 \mathrm{keV} \mathrm{Ar}^{+}$ions is shown with a single line at about $1.1 \mathrm{MeV}$ energy. This feature corresponds to the nuclear reaction $\mathrm{O}^{16}(\alpha, \alpha) \mathrm{O}^{16}$ [12]. The intensity of this line changes with the increase of energy of incident alpha particles. The highest intensity of this line is at the energy $3.045 \mathrm{MeV}$. The line disappears in the RBS/NR spectra measured at the incident $\alpha$ particles energy of $3.060 \mathrm{MeV}$. The results confirm the presence of a thin oxide layer on the implanted GaAs surfaces. The thicknesses of the oxide layers (between 4 and $7 \mathrm{~nm}$ ) were obtained on the basis of these measurements using the computer code SIMNRA [13].

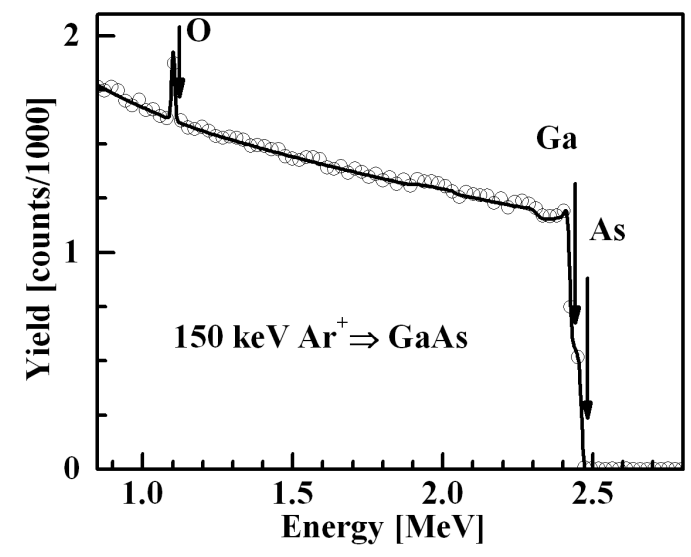

Fig. 1. RBS/NR spectrum of $\mathrm{Ar}^{+}$ion implanted GaAs at a fluence of $1 \times 10^{16} / \mathrm{cm}^{2}$.

Normalized yield of particles scattered on the nuclei of virgin and ion implanted GaAs in a vicinity of $2.45 \mathrm{MeV}$ is shown in Fig. 2. Close inspection shows that the detailed shape of the yield changes for GaAs implanted with

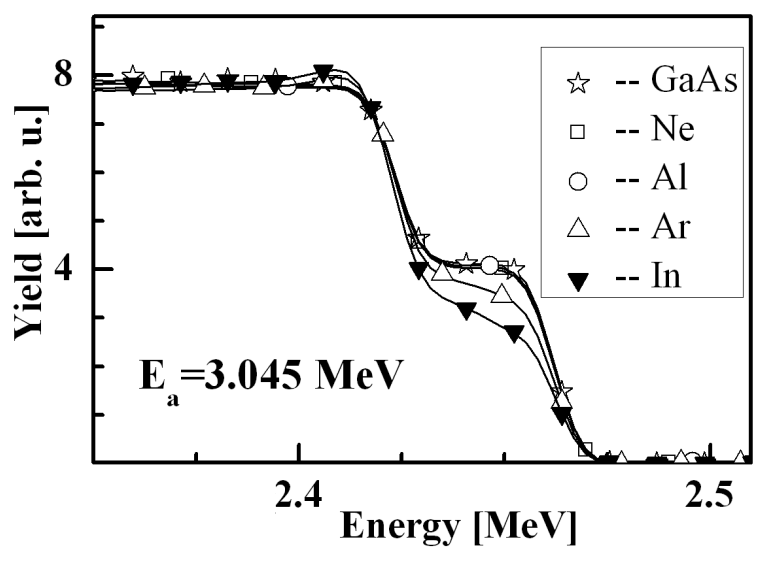

Fig. 2. Part of RBS spectra collected for virgin GaAs and GaAs implanted with different ions.

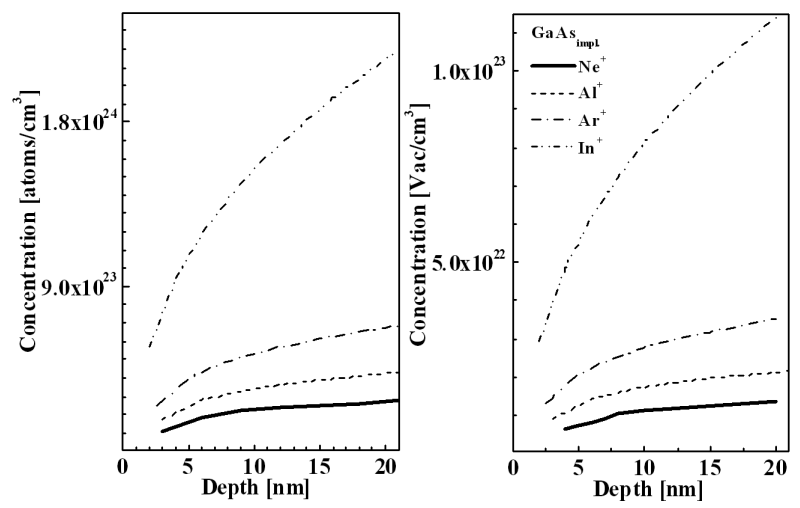

Fig. 3. Depth profiles of atoms and vacancies in near surface region of GaAs after implantation with different ions.

Ar and In ions. This is probably due to differences in diffusion process of As and Ga atoms during the implantation of various ion species. This process, along with the embedding of the implanted elements into GaAs causes a local change of the atomic density. These changes are associated with an increase in the concentration of vacancies formed in the near surface layers of implanted GaAs. The number of vacancies produced during implantation increases with the mass of ions (for the same ion fluence). The implanted atoms and vacancies depth distributions have been calculated for different ions by the SRIM code [14] and are presented in Fig. 3. This growth of disorder in the studied subsurface layers causes the intensification of diffusion processes of atoms from these layers to the irradiated surface. The first process is referred to diffusion of As and Ga. The coefficient of diffusion is lower for gallium. These values for As and $\mathrm{Ga}$ atoms in GaAs are: $5.5 \times 10^{-4} \mathrm{~cm}^{2} / \mathrm{s}$ and $4 \times 10^{-5} \mathrm{~cm}^{2} / \mathrm{s}$, respectively [15]. Atoms of As leave the disordered subsurface layers during the implantation faster than Ga. The second process is connected with the increase of the In and $\mathrm{Al}$ atomic concentrations in the subsurface layers. The same effects were observed in [16]. 


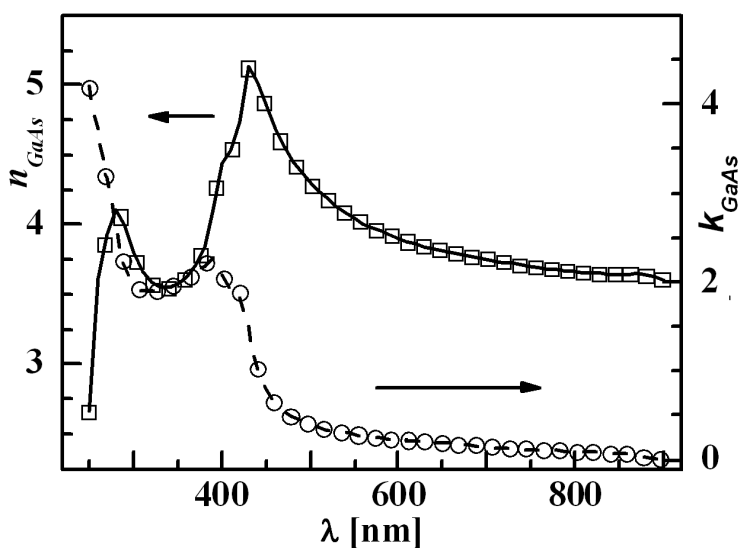

Fig. 4. Refraction index $n$ and extinction coefficient $k$ of GaAs. The points represent the measured spectra, lines are taken from [18].
Using the measurement values of ellipsometric (VASE) parameters $\Psi$ and $\Delta$ performed at 3 incidence angles: $65^{\circ}, 70^{\circ}$ and $75^{\circ}$, the refraction index $n$ and extinction coefficient $k$ spectra of unimplanted GaAs were obtained in the range from $250 \mathrm{~nm}$ to $900 \mathrm{~nm}$ (Fig. 4). The sample was approximated by the three-phase model (an ambient, a native oxide layer, and the substrate). The thickness of the native oxide layer was taken from the results of the RBS/NR investigations on the same sample. We used the refraction index and extinction coefficient spectra of native oxide on GaAs, calculated on the basis of the Zollner paper [17]. The obtained $n$ and $k$ spectra of GaAs (Fig. 4) are in a very good agreement with those from [18]. This is the evidence that the model which assumes the homogeneous oxygen-enriched layer on GaAs is appropriate and the thickness of the native oxide layer was determined accurately by the RBS/NR method.

Optical parameters of native oxide layers on ion-implanted GaAs.

TABLE

\begin{tabular}{c|c|c|c|c|c|c|c}
\hline \hline \multicolumn{2}{c|}{$\begin{array}{c}\text { GaAs } \\
\text { implanted with }\end{array}$} & \multicolumn{6}{c}{ Cauchy parameters } \\
\hline Ion & $\begin{array}{c}\text { Energy } \\
{[\mathrm{keV}]}\end{array}$ & $A$ & $\begin{array}{c}B \\
{\left[\mathrm{~nm}^{2}\right]}\end{array}$ & $\begin{array}{c}C \\
{\left[\mathrm{~nm}^{4}\right]}\end{array}$ & $k_{0}$ & $\begin{array}{c}\beta \\
{[\mathrm{nm}]}\end{array}$ & $\begin{array}{c}\gamma \\
{[\mathrm{nm}]}\end{array}$ \\
\hline $\mathrm{Ne}^{+}$ & 100 & 1.6005 & 0.0003 & $6.0 \times 10^{-4}$ & $2.30 \times 10^{-6}$ & 0.4394 & 443.18 \\
$\mathrm{Al}^{+}$ & 120 & 1.9315 & 0.0019 & $7.8 \times 10^{-6}$ & $2.10 \times 10^{-3}$ & 1.5164 & 150.40 \\
$\mathrm{Ar}^{+}$ & 150 & 2.0074 & 0.0025 & $1.0 \times 10^{-4}$ & $9.00 \times 10^{-3}$ & 0.4057 & 234.50 \\
$\mathrm{In}^{+}$ & 250 & 2.0954 & 0.0039 & $9.0 \times 10^{-4}$ & $5.26 \times 10^{-2}$ & 0.6731 & 529.35
\end{tabular}

For the calculation of the optical parameters of the native oxide layers on ion-implanted GaAs it was assumed that the refraction index spectrum of the oxide is described by the Cauchy equation [11]:

$$
n(\lambda)=A+\frac{B}{\lambda^{2}}+\frac{C}{\lambda^{4}}+\ldots
$$

where $A, B, C$ are the Cauchy coefficients and $\lambda$ stands for the wavelength in $\mathrm{nm}$.

The wavelength dependence of the extinction coefficient was fitted by the following curve [18]:

$$
k(\lambda)=k_{0} \mathrm{e}^{\beta\left(1240\left(\frac{1}{\lambda}-\frac{1}{\gamma}\right)\right)},
$$

where $k_{0}$ is the g mplitude, $\beta$ is the exponent factor, $\gamma$ is the band edge. This formula is based on the well-known Urbach law of exponential tail usually associated with localized states below a band gap in disordered solids [19]. Table contains the obtained values of fitting parameters for the oxide layers on ion-implanted GaAs samples. Using this information we calculated from the VASE ellipsometric angles the spectra of the real $\varepsilon_{1}$ and imaginary $\varepsilon_{2}$ parts of the native oxide dielectric function (Fig. 5). The results have shown that $\varepsilon_{1}$ values are the largest for oxide layers grown on GaAs implanted with the heaviest $\left(\mathrm{In}^{+}\right)$ions. The same is true for values of the imaginary $\varepsilon_{2}$ part of the dielectric function, which increase by a few

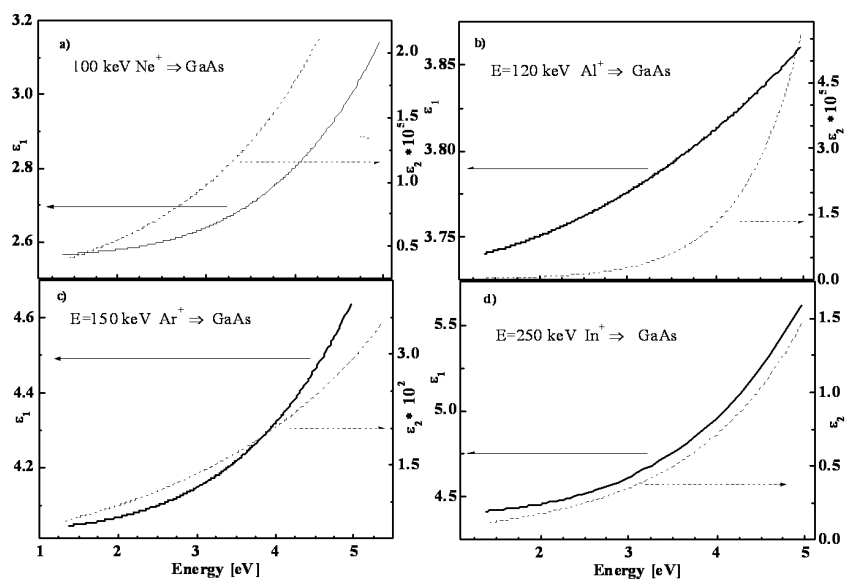

Fig. 5. Spectra of real and imaginary parts of dielectric function determined by VASE for native oxide layers on implanted GaAs.

orders of magnitude between the lightest investigated ion $\left(\mathrm{Ne}^{+}\right)$and $\mathrm{In}^{+}$.

\section{Conclusion}

Using ellipsometry we have obtained dielectric function spectra of the native oxide layers on SI (100) GaAs 
implanted with $\mathrm{Ne}^{+}, \mathrm{Al}^{+}, \mathrm{Ar}^{+}$, or $\mathrm{In}^{+}$ions at energies of $100,120,150$, and $250 \mathrm{keV}$, respectively. In calculations, the refraction index spectral dependence for native oxide was approximated with the Cauchy equations and extinction coefficient was fitted by the Urbach exponential formula. Complementary information on the oxide thickness was provided from the RBS/NR measurements. The investigations showed that both real and imaginary parts of the dielectric function increase very substantially with mass of the ion species used for implantation.

\section{References}

[1] J. Zhang, T.H. Kosel, D.C. Hall, P. Fay, IEEE Electron Dev. Lett. 29, 143 (2008).

[2] J.S. Williams, Mater. Sci. Eng. A 253, 8 (1998).

[3] W. Wesch, E. Wendler, T. Bachmann, O. Herre, Nucl. Instrum. Methods Phys. Res. B 96, 290 (1995).

[4] T. Lohner, M. Fried, N.Q. Khanh, P. Petrik, H. Wormeester, M.A. El-Sherbiny, Nucl. Instrum. Methods Phys. Res. B 147, 90 (1999).

[5] M. Kulik, J. Herec, J. Romanek, Vacuum 63, 761 (2001).

[6] W. Wesch, E. Wilk, K. Hehl, Phys. Status Solidi A 70, 243 (1982).

[7] M. Kulik, J. Zuk, A. Drozdziel, K. Pyszniak, F.F. Komarov, W. Rzodkiewicz, Mater. Sci. Eng. B $\mathbf{1 7 6}$, 340 (2011).
[8] W. Rzodkiewicz, M. Kulik, K. Pyszniak, A.P. Kobzev, Acta Phys. Pol. A 116, 129 (2009).

[9] M. Turek, S. Prucnal, A. Drozdziel, K. Pyszniak, Rev. Sci. Instrum. 80, 043304 (2009).

[10] M. Turek, S. Prucnal, A. Drozdziel, K. Pyszniak, Nucl. Instrum. Methods Phys. Res. B 269, 700 (2011).

[11] Guide to Using WVASE 32, Software for Vase and M44 Ellipsometers, J.A. Woollam Co., Lincoln (NE) 1991.

[12] J.R. Cameron, Phys. Rev. 90, 839 (1953).

[13] M. Mayer, SIMNRA User's Guide, www2.if.usp.br/ lamfi/guia-simnra.pdf, Max-Planck-Institut für Plasmaphysik, Garching 2012.

[14] J.F. Ziegler, SRIM - The Stopping and Range of Ions in Matter, http://www.srim.org (2009).

[15] Data in Science and Technology, Semiconductors, Group IV Elements and III- $V$ Compounds, Ed. O. Madelung, Springer-Verlag, Berlin 1991.

[16] M. Kulik, A.P. Kobzev, D. Jaworska, J. Zuk, J. Filiks, Vacuum 81, 1124 (2007).

[17] S. Zollner, Appl. Phys. Lett. 63, 2523 (1993).

[18] J.A. Woollam Co Inc., WVASE 32 program v. 3.441, tabulated at UNL, Nebrasca, USA.

[19] S.K.J. Al-Ani, K.I. Arshak, C.A. Hogarth, J. Mater. Sci. 19, 1737 (1984). 